\title{
Hologram recording in polyvinyl alcohol/acrylamide photopolymers by means of pulsed laser exposure
}

\author{
Celia Garcia, Inmaculada Pascual, Angel Costela, Inmaculada Garcia-Moreno, \\ Clara Gomez, Antonio Fimia, and Roberto Sastre
}

\begin{abstract}
The variation in transmittance produced when a photopolymer is irradiated with a pulsed laser is analyzed and experimental results obtained when diffraction gratings are stored using pulsed exposure are presented. In either case, the influence of the energy of the irradiation pulse, the number of pulses, and the pulse repetition rate were studied. The photopolymer used was an acrylamide/polyvinyl alcohol dry film with a yellow eosin-thiethanol-amine mixture as a photoinitiator system. The recording of the gratings was performed by use of a holographic copying process. The samples were exposed and holograms recorded with a collimated beam from a frequency-doubled Nd:YAG (532 nm) $Q$-switched laser. Our initial results show that it is possible to obtain diffraction gratings with a diffraction efficiency of $60 \%$ and a refractive index modulation up to $2.8 \times 10^{-3}$. The energetic sensitivities achieved are close to those obtained with the same material and continuous irradiation without a preprocessing of the gratings. (C) 2002 Optical Society of America
\end{abstract}

OCIS codes: $\quad 090.0090,090.2900,160.5470$.

\section{Introduction}

Holography is widely employed in the investigation of photochemical and photophysical processes by use of continuous-wave and pulsed-laser irradiation. The rate constants, quantum yields, and number of photons involved in reversible and irreversible photoprocesses are determined by studying the change in diffraction efficiency of gratings stored with continuous wave laser radiation. ${ }^{1}$

The phase-modulated holographic technique has enabled the quantum yield and the effects of a photoinduced matrix to be determined by monitoring am-

C. Garcia and I. Pascual are with the Universidad de Alicante, Departamento Interuniversitario de Optica, Apdo. 99, E-03080, Alicante, Spain. A. Costela, I. Garcia-Moreno, and C. Gomez are with the Consejo Superior de Investigaciones Cientificas, Instituto de Química-Física "Rocasolano," Serrano 119, E-28006 Madrid, Spain. A. Fimia is with the Universidad Miguel Hernández, Departamento de Ciencia y Tecnologia de Materiales. Division de Optica. Av. del Ferrocarril s/n. E-03202 Elche, Spain. R. Sastre is with the Consejo Superior de Investigaciones Cientificas, Instituto de Ciencia y Tecnologia de Polimeros, Juan de la Cierva 3, E-28006 Madrid, Spain. C. Garcia's e-mail address is c.garcia@ ua.es.

Received 27 August 2001; revised manuscript received 2 January 2002.

0003-6935/02/142613-08\$15.00/0

(C) 2002 Optical Society of America plitude and phase gratings. ${ }^{2}$ Carre et al. ${ }^{3}$ developed an alternative treatment, which makes it possible to obtain information from photochemical processes by use of the bleaching of the sensitizer in a grating formed by the holographic method.

Blaya et al., ${ }^{4}$ by combining the formation of volume holograms with the temporal evolution of transmittance, obtained a model that enables the polymerization constants of the radical polymerization mechanism involved during irradiation, the grade of conversion at any time, the quantum yield of the initiation of radicals, and the molar absorption of the dye to be determined.

The formation of dynamic holograms with ultrashort light pulses was analyzed in different holographic recording materials. ${ }^{5-7}$ In photorefractive materials ${ }^{5}$ the technique used combines a pulsed signal beam with a continuous reference beam. In this way the diffraction efficiency can be maximized and greatly increased in comparison with the traditional continuous recording technique. It was demonstrated that reflection holograms may be recorded in Dupont photopolymer films by means of pulsed-laser exposure, ${ }^{6}$ and it was observed that continuous incoherent preillumination increased both diffraction efficiency and sensitivity. The behaviour was also analyzed of acrylic-based photopolymers when exposed to intermittent or pulsed laser sources. ${ }^{8}$ In this case an Argon laser was used to study the ther- 
mal aftereffects in the holographic recording process, when the recording was realized by a laser beam with a single laser pulse, chopped repetitively with constant frequency and with variable frequency.

Furthermore, pulsed laser exposure was used for evaluation of photochemical rate constants and quantum yields. Olaj et al. ${ }^{9}$ developed the pulsed-laser polymerization method as a tool for evaluating individual kinetic constants of free-radical polymerization. The free-radical propagation rate coefficients of methyl methacrylate were measured with visible light wavelengths. ${ }^{10}$

Decker et al. ${ }^{11}$ studied the kinetics of the polymerization of an acrylic monomer exposed to a pulsed nitrogen laser tuned at $337.1 \mathrm{~nm}$ and determined the polymerization rate and the induction period. Deeg et al. ${ }^{12}$ demonstrated that it is possible to perform single-shot transient grating experiments, and thus, in principle, to investigate every photoinduced irreversible process. Unstable photochemical intermediates can be detected and their kinetics evaluated. Together with the quantum yield measured by a continuous-wave-grating technique, this allows the photochemical rate constants to be calculated.

In this paper we study the response of a polyvinyl alcohol/acrylamide photopolymer deposited in the form of a layer when it is exposed to pulsed-laser irradiation. When using pulsed laser beams with their inherent high intensity, two different aspects of the process have to be considered. First, the nonlinearity of the material's response, which clearly influences sensitivity and diffraction efficiency. Second, the discontinuity of irradiation, which makes it necessary to consider the mechanism of photopolymerization involved in the refractive-index modulation in a different way than under continuous-wave irradiation.

The photopolymerization begins by absorption of light by the photoinitiator, which results in the generation of primary free radicals. These radicals will be considered to be identical with respect to their reactivity. The second step in the initiation process is the true chain of initiation, in which primary radicals react with a monomer unit to form a growing radical of one repeat unit in length. After, in the propagation step, a monomer is added, following a chain-reaction process, to the growing polymer radical. Two separate paths exist for termination. The first is the normal bimolecular combination, in which two growing macroradicals come together and terminate. The second path for termination is disproportionate, in which a labile atom (usually hydrogen) is transferred from one polymer radical to another. Although the predominant reaction depends on the nature of the reactivity monomer as well as the temperature, the combination of growing polymer radicals should predominate in the present case as a consequence of both low temperature and the solid matrix employed.

Under pulsed irradiation this mechanism changes. The first laser pulse generates a population of free radicals. In the dark time between two consecutive pulses the radicals react with the monomer and the
Table 1. Formulation of the Photopolymeric System

\begin{tabular}{cl}
\hline Components $^{a}$ & Concentration \\
\hline TEA & $0.199 \mathrm{M}$ \\
AA & $0.446 \mathrm{M}$ \\
PVA & $10 \% \mathrm{w} / \mathrm{v}_{2} \mathrm{O}$ \\
YE & $2.5 \times 10^{-4} \mathrm{M}$ \\
\hline
\end{tabular}

${ }^{a}$ TEA, triethanolamine; AA, acrylamide; PVA, polyvinyl alcohol; YE, yellow eosin.

polymer chain grows. At the next laser pulse, a large population of primary radicals is produced again. These can react with the growing polymer chains causing premature termination. The surviving radicals can also react with the monomer during the next dark time and the process is repeated.

In this study we first analyze the variation in transmittance of the material as a function of both pulse fluence and repetition rate. ${ }^{13}$ This enables information to be obtained about the rate of polymerization as a function of these parameters. Second, we present the experimental results obtained when holograms are stored using pulsed beams. ${ }^{14}$ We also study the effect of both pulse fluence and total number of pulses employed during irradiation to obtain maximum diffraction efficiency.

The use of pulsed lasers makes it necessary to adjust carefully the optical paths to zero differences and to take into account the wider spectral band as compared with that of cw lasers. Thus the interest of using arrangements where irradiation is done by a copy process ${ }^{15,16}$ that is based on those conditions where the requirements of spatial and temporal coherence are less severe than in a conventional holographic system.

\section{Material}

A typical photopolymeric recording-material system is composed of a polymeric matrix that supports monomers and the photoinitiator system. The photopolymeric formulation used in this work is based on acrylamides. ${ }^{17}$ It consists of acrylamide as a monomer, triethanolamine as electron donor coinitiator, and yellowish eosin as a photoinitiator: all on a film of polyvinyl alcohol as a matrix.

The photosensitive solution is prepared by adding 2 $\mathrm{ml}$ of a $8 \mathrm{~g} / \mathrm{l}$ aqueous solution of yellowish eosin (supplied by Panreac), $6.4 \mathrm{ml}$ of a solution of acrylamide (supplied by Sigma), and triethanolamine (supplied by Sigma) to $40 \mathrm{ml}$ of $10 \% \mathrm{w} / \mathrm{v}$ polyvinyl alcohol (supplied by Riedel-de-Haen and a molecular weight $\approx 25,000$ ) in water. The concentration of the composition is summarized in Table 1.

The resulting solution is coated, using an automatic depositor, on a $20 \times 40 \mathrm{~cm}^{2}$ glass plate to give an initial thickness of $500 \mu \mathrm{m}$. The plate is dried for $24 \mathrm{~h}$ in the dark under normal laboratory conditions (temperature $\approx 21-23{ }^{\circ} \mathrm{C}$, relative humidity $\approx 50-$ $60 \%$ and subsequently cut into plates measuring $6.5 \times 6.5 \mathrm{~cm}^{2}$. The thickness of the layer was mea- 


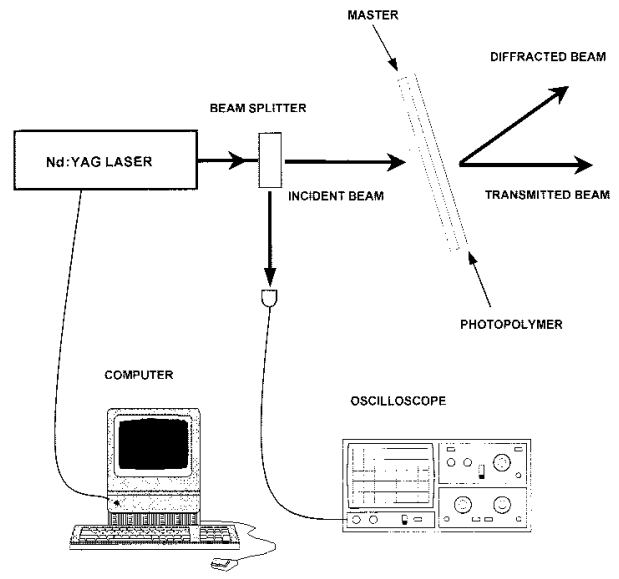

Fig. 1. Experimental setup.

sured with a PIG 455 apparatus supplied by Neurtek. The final thickness of the dry film is $70 \pm 5 \mu \mathrm{m}$.

\section{Experimental Setup}

First, the variation in transmittance of the material at the emission wavelength of the laser, $\lambda=532 \mathrm{~nm}$, as a function of the number of pulses and the fluence per pulse was analyzed. To do so, the laser beam was made to strike the photopolymer perpendicularly. The transmittance was measured with a Cary $3 \mathrm{E}$ spectrophotometer to obtain information about the photochemical behavior of the dye.

Figure 1 shows the geometry of the irradiation arrangement used to store the holograms by means of a pulsed beam. The process is a copying one, in which the master is placed in direct contact with the photopolymer in such a way that the transmitted and diffracted beams interfere in the photopolymer film, provided that the paths of the beams differ sufficiently to produce interference.

A beam splitter placed in the way of the pump beam directs about $10 \%$ of the laser beam energy toward a detector. The generated signal was a measure of the pump pulse energy and was recorded with an oscilloscope. The remaining $90 \%$ of the pulse energy is incident on the original pattern at an angle so that the diffracted and transmitted beams impinge onto the photopolymer film. A computer controls both the number and repetition rate of the pump pulses. The diffracted intensity was monitored with a He-Ne laser tuned at $633 \mathrm{~nm}$, at which wavelength the material does not absorb. Before measure the diffracted intensity the master is removed.

In a previous study we analyzed the coherence conditions of this geometry. ${ }^{15}$ If we consider that the master is a photographic emulsion $5-\mu \mathrm{m}$ thick and that the photopolymer has a thickness of $70 \mu \mathrm{m}$, applying the results of this previous study we obtain a difference in path of $1.69 \mu \mathrm{m}$, which gives a coherence factor of 0.99 . Because the spectral width of the pulse is $1 \mathrm{~nm}$, its temporal coherence factor is

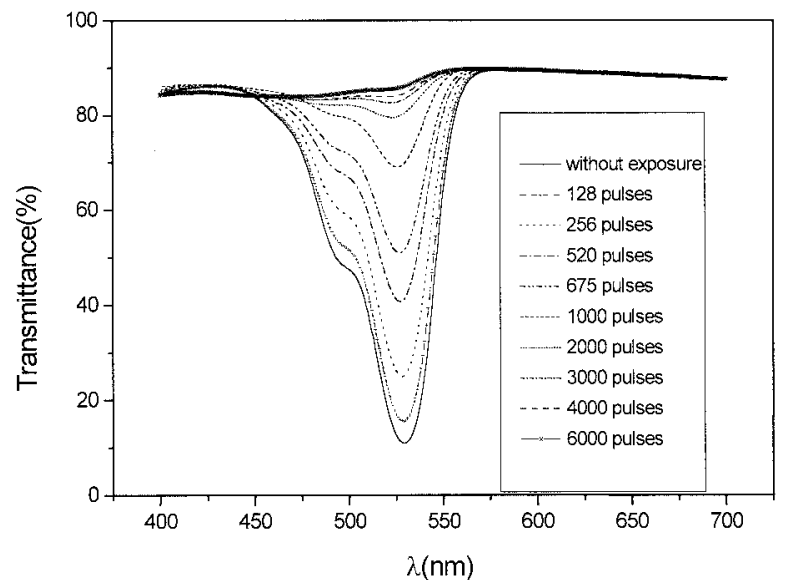

Fig. 2. Transmittance spectra for different number of pulses at a pulse fluence of $1.74 \mathrm{~mJ} / \mathrm{cm}^{2}$ and a pulse repetition rate of $3 \mathrm{~Hz}$.

almost one, so the total coherence factor is greater than 0.98 .

The original grating (master) is formed in a photographic emulsion processed in silver halide sensitized gelatin. The ratio of the transmitted beam to the diffracted beam is $1: 2$ and it has a total transmittance of $75 \%$, which gives a fringe visibility of 0.94 . These data indicate that it is not possible to obtain a diffraction efficiency of $100 \%$ with this geometry, because the phase contrast is not one. Nevertheless, from the point of view of the analysis and optimization of the photopolymer, the simplicity of the device compensates for this limitation in the diffraction efficiency.

We exposed the samples by recording holograms with a collimated beam from a frequency-doubled $\mathrm{Nd}$ : YAG (532 nm) $Q$-switched laser. The pulse duration was $\sim 8 \mathrm{~ns}$ and the repetition rate varied between 1 and $10 \mathrm{~Hz}$.

\section{Study of the Transmittance Evolution}

First, the variation in transmittance of the material as a function of the pulse repetition rate, the pulse fluence and the number of pulses was analyzed. In the presented experiments, the pulse fluence was increased from $0.07 \mathrm{~mJ} / \mathrm{cm}^{2}$ to $6.7 \mathrm{~mJ} / \mathrm{cm}^{2}$ and the pulse repetition rate was varied from $1 \mathrm{~Hz}$ to $10 \mathrm{~Hz}$.

Figure 2 represents the evolution of the transmittance spectra of the sample for a pulse fluence of 1.74 $\mathrm{mJ} / \mathrm{cm}^{2}$, irradiated with a pump repetition rate of 3 $\mathrm{Hz}$ and a different number of pulses. As can be seen, the variation in transmittance with the number of pulses is nonlinear: when this parameter increases the transmittance first increases. However, up to 2000 pulses the variation in transmittance seems to be least dependent on the number of pump pulses. This behavior is expected and directly related with the depletion of the photoinitiator and thus with the absorbance reached at the different number of pump pulses.

Figure 3 shows the variation in transmittance of the material at the emission wavelength of the la- 
ser, $\lambda=532 \mathrm{~nm}$, normalized to the transmittance line of the unexposed plate, as a function of the number of pulses for different pulse fluences. This variation in transmittance indicates that the material is bleached when it is illuminated with light of $532 \mathrm{~nm}$, which corresponds to the absorption band of yellowish eosin. Therefore incident light produces consumption of the sensitizer caused by a photoreduction reaction of the dye, giving rise to the formation of the alkyl-amino free radicals that initiate the polymerization reaction. When the fluence per pulse is increased, the variation in transmittance for a given number of pulses increases, which implies a greater consumption of the dye and, consequently, an increase in the rate of generation of free radicals.

The curves can be fitted by using an approximation that can be easily obtained from the experimental characteristics of the holographic film. ${ }^{18}$ We considered the formula:

$$
\Delta T=\frac{T_{\infty}}{1+\exp (a-b \log E)+\exp (c-d \log E)},
$$

where $\Delta T$ is the variation in transmittance, $T_{\infty}$ is the maximum variation in transmittance, $E$ is the exposure, $a$ and $b$ are parameters that can be determined from the experimentally obtained $\Delta T$ $\log E$ curve, and $\mathrm{c}$ and $\mathrm{d}$ can be estimated by evaluating Eq. (1) at two points $\log \mathrm{E}=\log E i$ and $\log E=(\log E i) / 2$. If $E i$ is the exposure of the inertial point and $m$ is the slope of the characteristic curve in the linear region, from simple calculation it can been obtained ${ }^{18}$

$a=2 \frac{1+2 m \log E_{i}}{T_{\infty}}$,

$b=\frac{4 m}{T_{\infty}}$,

$c=$

$\ln \left(\frac{\left\{\frac{\Delta T_{\infty}}{\Delta T\left(\frac{1}{2} \log E_{i}\right)}-\left[1+\exp \left(2+\frac{2 m \log E_{i}}{\Delta T_{\infty}}\right)\right]\right\}^{2}}{\frac{\Delta T_{\infty}}{\Delta T\left(\log E_{i}\right)}-(1+\exp 2)}\right.$,

$d=\frac{c-\ln \left[\frac{\Delta T_{\infty}}{\Delta T\left(\log E_{i}\right)}-(1+\exp 2)\right]}{\log E_{i}}$.

By means of numerical fitting, we can determine the slope of the linear zone together with the minimum

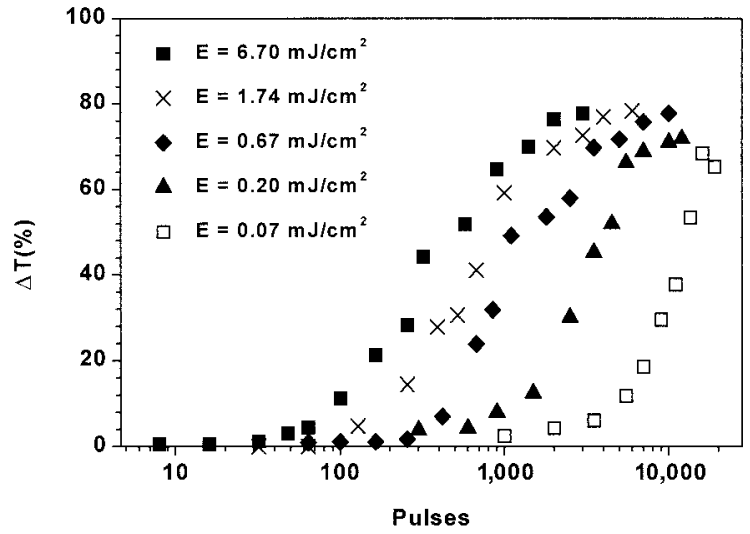

Fig. 3. Variation in transmittance as a function of the number of pump pulses for different laser fluences.

activation energy of the dye necessary to initiate radical generation.

As an example, experimental points and fitted curves are shown in Fig. 4 for the fluences of two pulses, $0.67 \mathrm{~mJ} / \mathrm{cm}^{2}$ and $1.74 \mathrm{~mJ} / \mathrm{cm}^{2}$. As can be seen from this figure, there is good agreement between theoretical and experimental results.

Figure 5 shows the slope obtained and the activation energy as a function of the pulse fluence. In the low fluence regime, when the pulse fluence increases, there is a decrease in both the slope and the activation energy. Above $0.67 \mathrm{~mJ} / \mathrm{cm}^{2}$ the slope remains apparently constant and the activation energy increases, which means that although the rate of consumption of the dye is independent of the pulse fluence, when the fluence of the pulse is increased, a higher energy is needed to activate the system and, consequently, to initiate the polymerization reaction. These results are important because they indicate the rate of variation of the dye concentration into the sample and therefore the rate of generation of radicals and the minimum energy necessary in each case.

Another factor that affects the response of the material is the pulse repetition rate. In the theoretical models that explain the polymerization under continuous irradiation, a stationary state is assumed for the production and consumption of radicals involved in the polymerization reaction. Therefore, because at $t=0$ the concentration of radicals is 0 , a minimum time is necessary to reach the stationary state. Under pulsed irradiation, the generation of free radicals is discontinuous. Consequently, the time with no irradiation of the sample is important, because the number of radicals decreases during this time and so does the rate of polymerization.

At low repetition rates, the dark period is long enough for the free-radical concentration to drop to near zero, and the polymerization rate is much lower than that reached under continuous irradiation. If the repetition rate increases so that this decay is incomplete during the dark phase and the steadystate concentration is not reached during the illumination phase, the radical concentration becomes much higher and consequently increases the rate of 

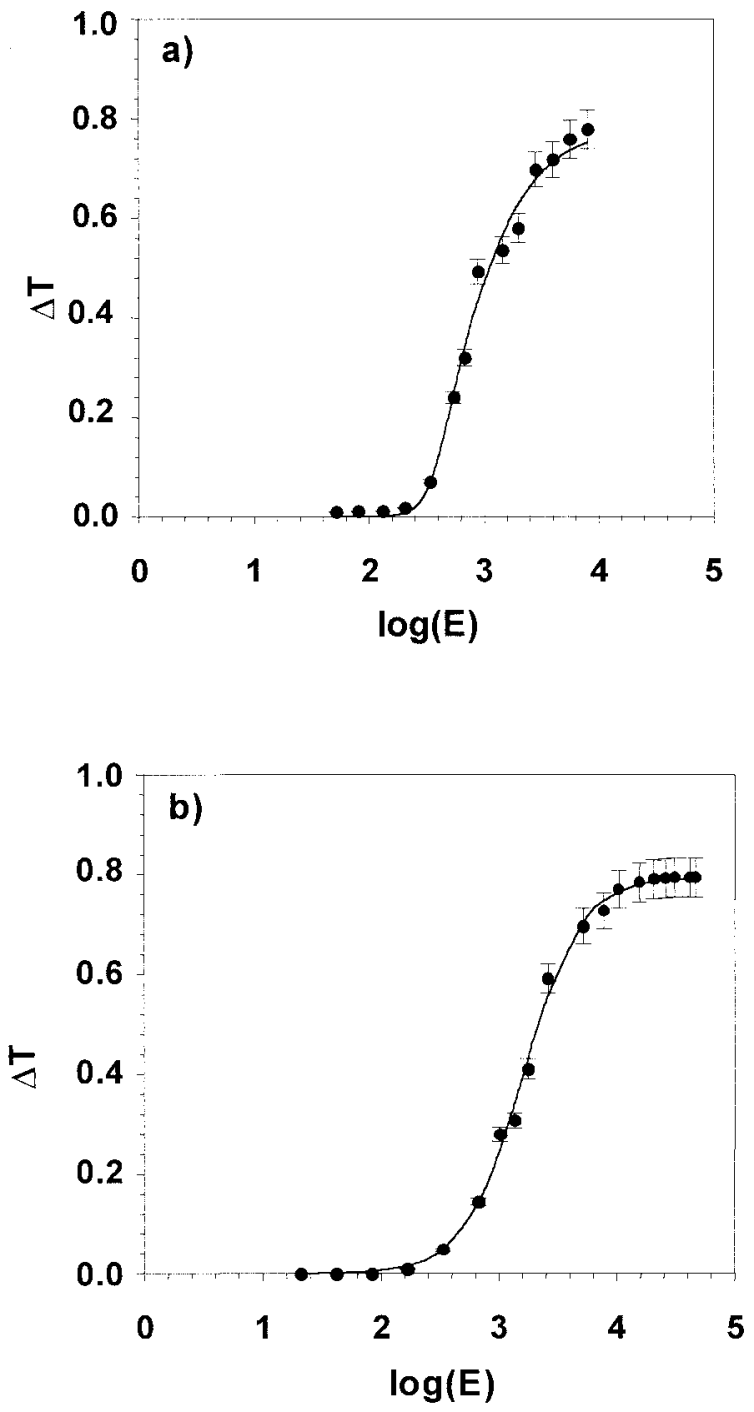

Fig. 4. Experimental and theoretical transmittance curves as a function of log of exposure for two pulse fluences: a) $0.67 \mathrm{~mJ} / \mathrm{cm}^{2}$ and b) $1.74 \mathrm{~mJ} / \mathrm{cm}^{2}$.

polymerization. At repetition rates high enough, the free-radical concentration remains near constant and the conditions are similar to those reached under continuous irradiation. This behaviour is illustrated in Fig. 6, which shows the evolution of the transmittance function versus the pulse repetition rate for a pulse fluence of $2.2 \mathrm{~mJ} / \mathrm{cm}^{2}$ and 1000 pulses. As can be appreciated, the variation in transmittance increases when the pulse repetition rate is increased until a certain value $(\approx 5 \mathrm{~Hz})$ is reached, after which it remains constant. This behavior can be explained by the so-called saturation effect, which is not specifically induced under laser irradiation, because the same was observed when laser irradiation was operated at very large and focused high light intensities. The most likely reason for this saturation effect is that once the concentration of the excited photoinitiator reaches a certain high value, it partially recombines to render again the starting dye. Because chain initiation and chain

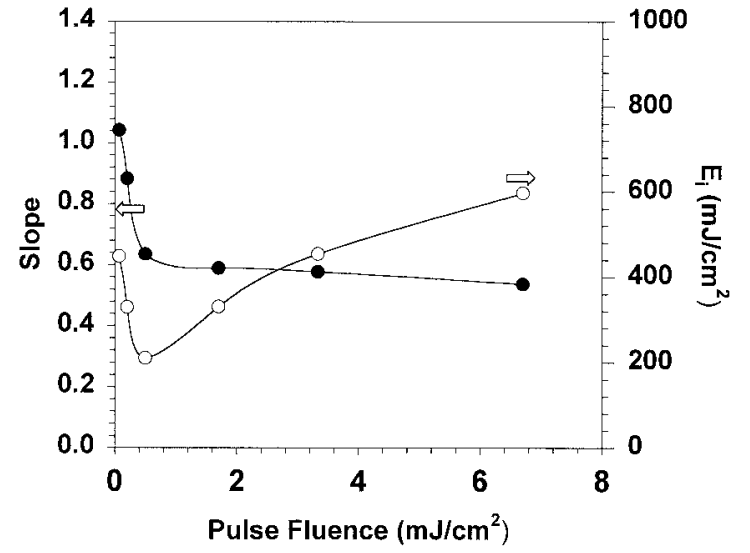

Fig. 5. Slope of linear region (filled circles) and the activation energy (open circles) as a function of the laser pulse fluence for a repetition rate of $3 \mathrm{~Hz}$. Solid curves are just to help the eyes.

growth is expected to be controlled by diffusion, the observed saturation effect or the reversibility of part of the photoinitiator depleted should be favored in the present case, taking into account that we are working in a solid polyvinyl alcohol matrix. ${ }^{19}$

\section{Storage of Diffraction Gratings}

As mentioned above, a copying process was used to generate the holograms. Figure 7 shows diffractionefficiency results of the +1 order in the case of different fluences per pulse, measured with an $\mathrm{He}-\mathrm{Ne}$ laser tuned at $633 \mathrm{~nm}$, at which wavelength the material does not absorb. Each point in the figure corresponds to increased numbers of irradiation pulses whose individual energies are added together. It can be seen that diffraction efficiencies of $55 \%$ have been reached for a pulse fluence of $0.67 \mathrm{~mJ} / \mathrm{cm}^{2}$. The sensitivity of the material in this case $(235 \mathrm{~mJ} /$ $\mathrm{cm}^{2}$ ) is similar to that obtained with continuous irradiation. ${ }^{17}$ However, the diffraction efficiency decreases from $80 \%$ for continuous irradiation to $55 \%$ for irradiation with a pulsed laser. There is an optimum fluence per pulse $\left(0.67 \mathrm{~mJ} / \mathrm{cm}^{2}\right)$ at which maximum sensitivity (defined as the minimum energy

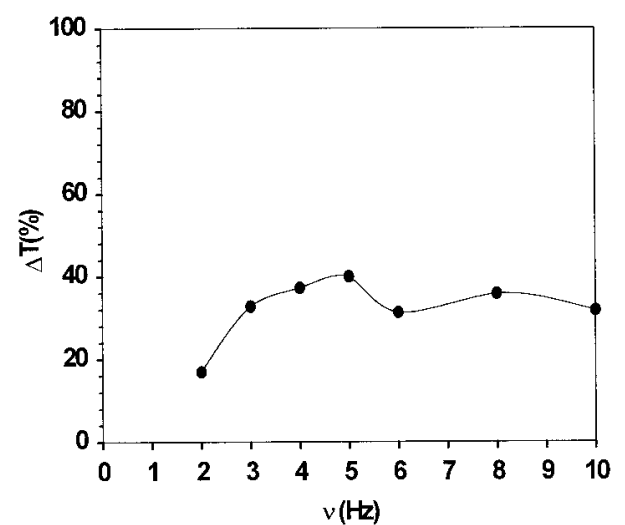

Fig. 6. Variation in transmittance as a function of the pulse repetition rate for a pulse fluence of $2.2 \mathrm{~mJ} / \mathrm{cm}^{2}$ and 1000 pulses. Solid curve is just to help the eyes. 


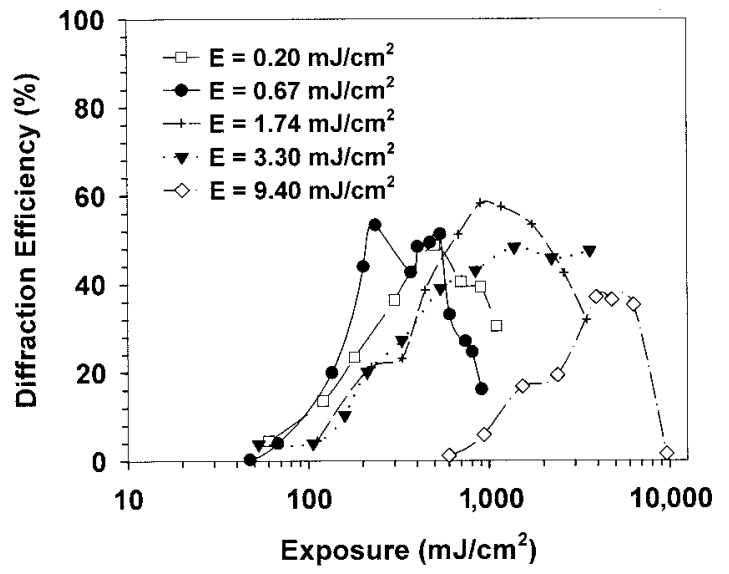

Fig. 7. Diffraction efficiency as a function of exposure for different pulse fluences and a repetition rate of $10 \mathrm{~Hz}$. The points correspond to an increased number of irradiation pulses.

necessary to reach maximum diffraction efficiency) and maximum diffraction efficiency are obtained. For laser fluences below $0.67 \mathrm{~mJ} / \mathrm{cm}^{2}$, lower diffraction efficiencies and sensitivities are obtained, while for laser fluences greater than $0.67 \mathrm{~mJ} / \mathrm{cm}^{2}$ the sensitivity decreases.

From the figure it may be concluded that a minimum number of pulses is necessary to activate the system and to store a grating with a measurable diffraction efficiency. The number of pulses necessary depends on the incident intensity. We found that for $0.2 \mathrm{~mJ} / \mathrm{cm}^{2}$, the number is 300 ; for 0.67 $\mathrm{mJ} / \mathrm{cm}^{2}, 100$; for $1.74 \mathrm{~mJ} / \mathrm{cm}^{2}, 64$; for $3.3 \mathrm{~mJ} / \mathrm{cm}^{2}$, 16 , and for $9.4 \mathrm{~mJ} / \mathrm{cm}^{2}, 64$.

If we calculate the maximum refractive-index modulation starting from the diffraction-efficiency curves for different fluences, we determine that for $0.2 \mathrm{~mJ} /$ $\mathrm{cm}^{2}$, the refractive index modulation is approximately $2 \times 10^{-3}$; for $0.67 \mathrm{~mJ} / \mathrm{cm}^{2}, 2.3 \times 10^{-3}$; for 1.74 $\mathrm{mJ} / \mathrm{cm}^{2}, 2.5 \times 10^{-3}$, and for $9.4 \mathrm{~mJ} / \mathrm{cm}^{2}, 2 \times 10^{-3}$. Taking into account that under continuous irradiation the refractive-index modulation achievable is approximately $3 \times 10^{-3}$, the values we obtained with pulsed irradiation are close to those obtained with continuous irradiation.

If we compare Figs. 3 and 7 for the same fluence
(Fig. 8), it can be seen that in order to obtain maximum diffraction efficiency, it is not necessary for the dye to be completely consumed. In fact, the consumption of dye necessary to reach the maximum diffraction efficiency depends on the pulse fluence. As occurs with the diffraction efficiency curves (Fig. $7)$, there is an optimum pulse fluence $\left(0.67 \mathrm{~mJ} / \mathrm{cm}^{2}\right)$ at which the maximum diffraction efficiency is reached with the lowest variation in transmittance (5\%) [see Fig. 8(b)]. At lower and higher fluences [figures 8(a) and 8(c), respectively] the variation in transmittance at which the peak efficiency in diffraction is reached increases.

Another factor that influences the response of the material is the concentration of the dye. Figures 9 (a) and 9(b) represent the maximum diffraction efficiency and sensitivity, respectively, as a function of the pulse fluence for three different dye concentrations $\left(1.2 \times 10^{4} \mathrm{M}, 2.5 \times 10^{-4} \mathrm{M}\right.$, and $\left.5 \times 10^{-4} \mathrm{M}\right)$. When the concentration of the dye is increased, a higher fluence is needed to store the gratings with an appreciable diffraction efficiency. This coincides with the studies achieved with the technique of pulsed-laser polymerization. ${ }^{20}$ In these studies, when the initiator concentration is increased its quantum efficiency decreases, and under high initiator concentrations, quantum yields are actually reduced. When the pulse fluence is increased, the concentration of the dye is not so determinant in response to the material: for $3.3 \mathrm{~mJ} / \mathrm{cm}^{2}$ and 9.4 $\mathrm{mJ} / \mathrm{cm}^{2}$ the same diffraction efficiency is obtained for all concentrations. Concerning the sensitivity, upon increasing the concentration, the energy necessary to obtain the maximum diffraction efficiency increases, owing to the fact that its quantum efficiency decreases and the system becomes slower.

Although we have only carried out experiments for three different concentrations of the dye, the behavior exhibited by both the diffraction efficiency and sensitivity can be explained as follows: It is well known that in photopolymerization processes, the number of initiating radicals produced per unit of time ( $\mathrm{Ri})$ is directly related to the amount of photons absorbed, which depends, among other fac-
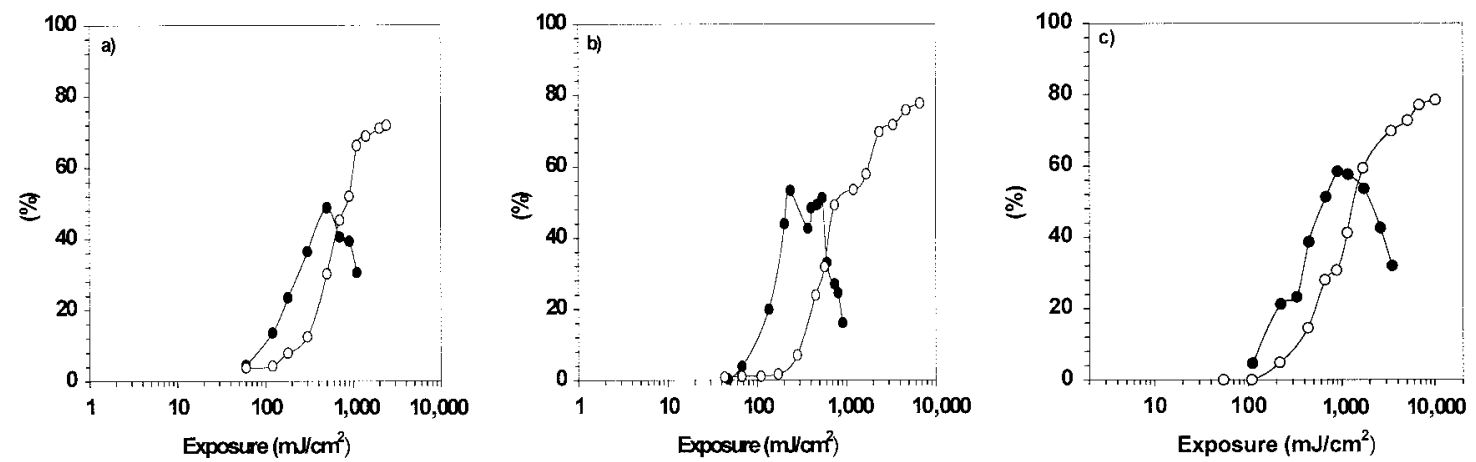

Fig. 8. Transmittance (open circles) and diffraction efficiency (filled circles) versus the exposure for a pulse fluence of: a) $0.2 \mathrm{~mJ} / \mathrm{cm}^{2}$, b) $0.67 \mathrm{~mJ} / \mathrm{cm}^{2}$, and c) $1.7 \mathrm{~mJ} / \mathrm{cm}^{2}$ and a repetition rate of $10 \mathrm{~Hz}$. Solid curves are just to help the eyes. 

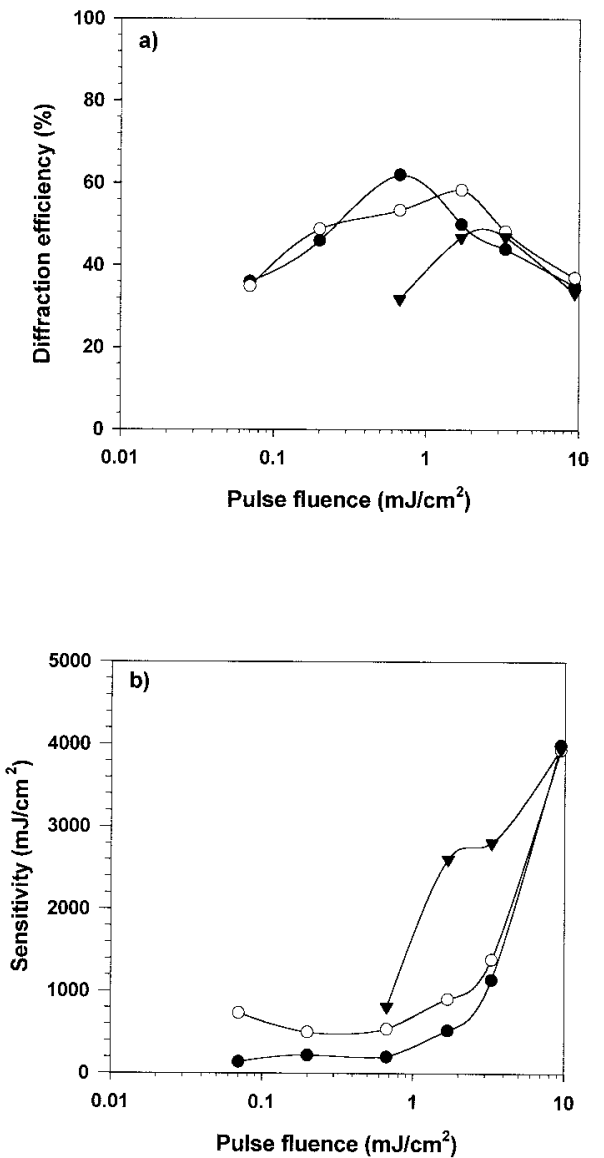

Fig. 9. a) Maximum diffraction efficiency and b) sensitivity as a function of pulse fluence for three different dye concentrations: $1.2 \times 10^{-4} \mathrm{M}$ (filled circles), $2.5 \times 10^{-4} \mathrm{M}$ (open circles), and $5 \times$ $10^{-4} \mathrm{M}$ (filled triangles). Solid curves are just to help the eyes.

tors, on the photoinitiator concentration (PI). Following Eq. 6:

$$
\mathrm{Ri}=\mathrm{I}_{0}\left[1-10^{-\mathrm{\epsilon l}(\mathrm{PI})}\right] \phi_{1} .
$$

$\mathrm{I}_{0}$ is the incident light intensity, $\epsilon$ is the molar extiction coefficient, $\mathrm{L}$ is the film thickness or the optical path length, and $\phi_{1}$ is the initiation quantum yield. Owing to this exponential law, the radicals will not be formed uniformly throughout the film, but mostly in the upper layer, particularly at high PI concentrations, as in the present case.

For a given light absorbance (A) of the film, the penetration of the laser beam $(\mathrm{L})$ will decrease as the photoinitiator concentration increases, according to a reciprocal law, namely:

$$
\mathrm{L}=\frac{\mathrm{A}}{\epsilon(\mathrm{PI})} .
$$

As expected, the photopolymerization rate increases rapidly with increasing PI concentration, but it tends to level off at high PI values, due to the internal filtering effect of the dye. The reduced penetration of light into the sample ultimately leads to a decrease in the overall rate of polymerization. ${ }^{21}$ Conse-

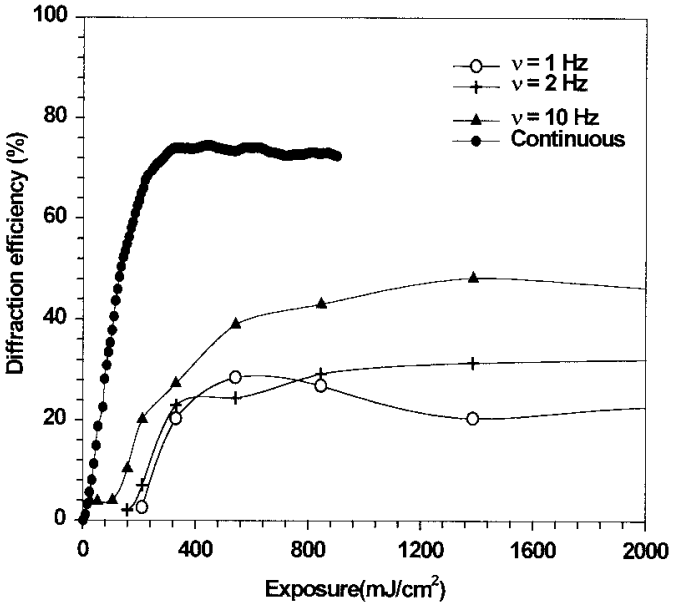

Fig. 10. Diffraction efficiency as a function of exposure for both continuous and pulsed irradiation with a laser fluence of $3.3 \mathrm{~mJ} /$ $\mathrm{cm}^{2}$ and a different repetition rate. Solid curves are just to help the eyes.

quently, the top layer will be highly polymerized, while the bottom layer remains unaffected.

As discussed previously, the laser repetition rate has an important effect on the response of the material. In particular, it exerts a direct influence on the molecular weight of the polymer formed.9,20 Olaj et al. determined that the chain length $\left(\mathrm{L}_{\mathrm{O}}\right)$ is given by

$$
\mathrm{L}_{\mathrm{O}}=k_{p}(\mathrm{M}) \mathrm{t}_{\mathrm{O}}
$$

where $t_{O}$ is the time between two laser pulses, $M$ is the monomer concentration, and $k_{p}$ is the propagation-rate coefficient.

At low laser repetition rates, the system behaves as if there were a single pulse, and a broad molecularweight distribution results that increases the chain length of the polymer formed. When the pulse repetition rate is increased, the free-radicals concentration increases and the possibility of premature termination is higher: Therefore the molecularweight distribution and the chain length of the polymer formed are reduced.

Figure 10 shows the energetic evolution of the diffraction efficiency for different repetition rates, 1,2 , and $10 \mathrm{~Hz}$ and a pulse fluence of $3.3 \mathrm{~mJ} / \mathrm{cm}^{2}$. As can be seen, the diffraction efficiency reached at high repetition rates is greater than that obtained at low ones, and the sensitivity decreases as this laser parameter increases.

Continuous irradiation is equivalent to work with an infinite repetition rate: Therefore the diffraction efficiency would have to be maximum and the sensitivity would have to be minimal. This is observed in Fig. 10, where the diffraction efficiency curves with pulsed irradiation are compared with the energetic evolution of the diffraction efficiency for continuous irradiation. In addition, as can be seen, the slope of the linear region increases with the laser repetition rate.

Finally, it should be noted that at high exposures 
noise gratings have been observed, which are characteristic of a system with a high temporal and spatial coherence.

\section{Conclusions}

The response to pulsed irradiation of a photopolymer of PVA/Eosin/Acrylamide has been analyzed and a high diffraction efficiency and sensitivity, close to those obtained with the same material under continuous irradiation, have been obtained. The experimental results obtained demonstrate the dependence of these parameters with the pulse fluence and the irradiation repetition rate. The variation of transmittance with the pulse energy is clearly nonlinear. A mathematical function, obtained from theoretical considerations, fits the experimental results obtained and allows us to find the dependence between slope and pulse energy.

High dye concentrations decrease the diffraction efficiency and sensitivity. The failure of the reciprocity law is related, in this case, to the high intensity of the irradiation, allowing us to conclude that the concentration used for pulsed irradiation must be lower than that selected for continuous irradiation. Experimental results of the diffraction efficiency as a function of the number of pulses indicate that a minimum number of pulses is necessary to activate photochemical reactions.

This work was financed by the Comisión Interministerial de Ciencia y Tecnología of Spain (Project MAT 2000-1361-C04-04 and Project MAT 99-0622).

\section{References}

1. D. M. Burland, "Applications of Holography in the Investigation of Photochemical Reactions," Acc. Chem. Res. 16, 218-224 (1983).

2. J. Pinsl, M. Gehrtz, and Chr. Bräuchle, "Phase-modulated holography: a new technique for investigation of solid-state photochemistry and hologram formation mechanism," J. Phys. Chem. 90, 6754-6756 (1986).

3. C. Carre, D. J. Lougnot, and J. P. Fouassier, "Holography as a Tool for Mechanistic and Kinetic Studies of Photopolymerization Reactions: A Theorical and Experimental Approach," Macromolecules 22, 791-799 (1989).

4. S. Blaya, L. Carretero, R. Mallavia, A. Fimia, and R. F. Madrigal, "Holography as a technique for the study of photopolymerization kinetics in dry polymeric films with a nonlinear response," Appl. Opt. 38, 955-962 (1999).

5. G. Li, L. Liu, B. Liu, and Z. Xu, "High-efficiency volume hologram recording with a pulsed signal beam," Opt. Lett. 23, 1307-1309 (1998).

6. K. T. Weitzel, U. P. Wild, V. N. Mikhailov, and V. N. Krylov, "Hologram recording in Dupont photopolymer films by use of pulse exposure," Opt. Lett. 22, 1899-1901 (1997).
7. A. V. Aristov, Yu E. Burunkova, D. A. Kozlovskii, and A. B. Nikolaev, "Pulsed recording of holograms by stepwise quantum excitation of media based on organic dyes," J. Opt. Technol. 66, 383-386 (1999).

8. D. J. Lougnot and C. Turck, "Photopolymers for holographic recording: III. Time modulated illumination and thermal post-effect," Pure Appl. Opt. 1, 269-279 (1992).

9. O. F. Olaj, I. Bitai, and F. Hinkemann, "The laser-flashinitiated polymerization as a tool of evaluating (individual) kinetic constants of free-radical polymerization, 2. The direct determination of the rate constant of chain propagation," Makromol. Chem. 188, 1689-1702 (1987).

10. M. D. Zammit, T. P. Davis, and G. D. Willet, "Visible light pulsed-laser polymerization at $532 \mathrm{~nm}$ employing a julolidine dye photosensitizer initiation system," Macromolecules 30, 5655-5659 (1997).

11. C. Decker and K. Moussa, "Kinetic investigations of photopolymerizations induced by laser beams," Makromol. Chem. 191, 963-979 (1990).

12. F. W. Deeg, J. Pinsl, and C. Bräuchle, "New Gratings Experiments in the Study of Irreversible Photochemical Reactions," IEEE J. Quantum Electron. QE-22, 1473-1481 (1986).

13. C. Garcia, A. Costela, A. Fimia, I. García-Moreno, I. Pascual, and R. Sastre, "Recording of diffraction gratings in polyvinyl alcohol/acrylamide photopolymers by pulsed laser irradiation," in Practical Holography XV and Holographic Materials VII, S. A. Benton, S. H. Stevenson, and T. J. Trout, eds., Proc. SPIE 4296, 274-281 (2001).

14. C. García, I. Pascual, A. Costela, I. García-Moreno, A. Fimia, and R. Sastre, "Experimental study of the acrylamide photopolymer with a pulsed laser," Opt. Commun. 188, 163-166 (2001).

15. I. Pascual, A. Beléndez, and A. Fimia, "A two-step method for recording holographic optical elements with partially coherent light," J. Opt. 22, 135-140 (1991).

16. I. Pascual, A. Beléndez, and A. Fimia, "Holographic system for copying holograms by using partially coherent light," Appl. Opt. 31, 3312-3318 (1992).

17. C. García, A. Fimia, and I. Pascual, "Holographic Behavior of a photopolymer at high thicknesses and high monomer concentrations: mechanism of photopolymerization," Appl. Physics B 72, 311-316 (2001).

18. H. Kasprzak, N. Sultanova, and H. Podbielska, "Nonlinear effects of the recording material on the image quality of a Fourier hologram," J. Opt. Soc. Am. A 4, 843-846 (1987).

19. C. Decker, "Laser curing, imaging, and polymerization kinetics" in Chemistry \& Technology of UV and EB Formulations (Wiley-Site Series, London, 1996) Vol. V, Chap. 4.

20. M. L. Coote, M. D. Zammit, and T. P. Davis, "Determination of free-radical rate coefficients using pulsed-laser polymerization," Trends Polym. Sci. 4, 189-196 (1996).

21. A. Costela, I. Garcia-Moreno, J. Dabrio, and R. Sastre, "Photochemistry and photopolymerization activity of p-nitroaniline in the presence of n,n-dimethylaniline as a bimolecular photoinitiator system," J. Polym. Sci. Part A Polym. Chem. 35, 3801-3812 (1997). 